\title{
Influencia de variables contextuales en el enfoque de enseñanza: relación entre enfoque de enseñanza y estilo de aprendizaje
}

Juan Puiggalí

Universidad de Girona, España

Email: joan.puiggaliaudg.edu

ORCID: 0000-0003-0647-5317

\author{
Montse Tesouro \\ Universidad de Girona, España \\ Email: montse.tesourodudg.edu \\ ORCID: 0000-0003-0704-4615
}

\section{RESUMEN}

A menudo los docentes no son conscientes de que el proceso de aprendizaje se ve afectado por diferentes factores. Consecuentemente, en este estudio se pretende analizar la percepción de los estudiantes sobre la influencia de variables contextuales en el enfoque de enseñanza (EE) utilizado por el docente y observar si existe relación entre este y el estilo de aprendizaje del estudiante (EA). Se han aplicado 2 cuestionarios: el apartado B-Approaches to studying del ASSIST y uno ad-hoc sobre EE. Contestaron ambos cuestionarios 1252 estudiantes de la Universidad de Girona. Los resultados muestran que la modalidad de estudios, tipo de asignatura y curso afectan en el EE, así como éste también afecta al EA. En los estudios de Humanidades y Ciencias Sociales, así como en los cursos más bajos ( $1^{\circ}$ y $2^{2}$ ) y en las asignaturas más prácticas (disponen de clases de problemas y/o prácticas de laboratorio) los profesores tienden a utilizar un EE centrado en el alumno. Se concluye que un profesor puede adaptar su EE a variables contextuales y que influye en la adopción del EA del estudiante: los que presentan un estilo profundo mayoritariamente indican que el profesor presenta un enfoque centrado en el alumno.

Palabras clave: Estilo de enseñanza, Estrategias de aprendizaje, Enseñanza superior, Efecto de contexto.

\section{Influence of contextual variables on the teaching approach: relationship between teaching approach and learning style}

\begin{abstract}
Teachers are often unaware that the learning process is affected by different factors. Consequently, the study aims to observe how contextual variables influence the teaching approach (TA) used by teachers and to see if there is a relationship between the students's learning style and the teacher's approach to teaching. Two questionnaires have been used: The B-ASSIST Approaches to studying the section and an ad-hoc questionnaire to learning approaches. 1,252 students from the University of Girona answered both questionnaires. The results show that the study type, the type of subject and course affect the TA. Similarly, the TA affects the student's learning style. In Humanity and Social Science studies, as well as in the lower courses (1st and 2nd) and the more practical subjects (they have classes of problems and/or laboratory practices), the teachers tend to use a student-centered approach. Finally, it is concluded that a teacher does not always use the same type of approach, because he can adapt to contextual variables and the TA used by the teacher influences the adoption of the student's learning style. In general students who have a deep learning style indicate that the teacher has a student-centered approach.
\end{abstract}

Key words: Teaching styles, Learning Strategies, Higher Education, Context effect. 


\section{Introducción}

Durante estas últimas décadas se han ido realizando diferentes estudios sobre los enfoques de enseñanza (EE) utilizados por los profesores al impartir docencia y sobre los estilos de aprendizaje (EA) que utilizan los estudiantes. No obstante, se ha realizado muy poca investigación sobre la gran influencia que tienen las variables contextuales como la modalidad de estudio, el curso, el tipo de asignatura... en el enfoque de enseñanza.

Se ha visto que el proceso de aprendizaje se ve afectado por diferentes factores (Özyurt y Özyurt, 2015). Por ejemplo, Frenzel (2014) señala que el estado de ánimo influye en cómo el docente imparte sus clases. En el marco de los EE, Rosário, Núñez, Valle, Paiva y Polydoro (2013) comentan que las investigaciones principalmente se han realizado en el contexto universitario y están orientadas al análisis de la relación entre los EE y variables contextuales. También existen estudios como los de Stes, Gijbels y Van Petegem (2008) que no encontraron que la asignatura impartida y el tamaño del aula influenciaran; Prosser, Ramsden, Trigwell y Martin (2003) que hallaron relación entre la experiencia docente y el EA utilizado; Tesouro, Cañabate y Puiggalí (2014) que mostraron una relación entre la modalidad de estudio y el EA, entre otros.

El diagnóstico y análisis de los EA resultan importantes para llegar a un aprendizaje efectivo ya que se puede mejorar la acción didáctica al ajustar el EE al EA (Soflano, Connolli y Haney, 2015).

En la presente investigación se pretende observar la influencia de las variables contextuales en el enfoque de enseñanza utilizado por el docente y observar si existe relación entre el estilo de aprendizaje utilizado por el alumno y el enfoque de enseñanza del docente.

\subsection{Enfoques de enseñanza}

En la década de los 90, dentro del ámbito de la educación universitaria, algunos autores como Trigwell y Prosser (1996) desarrollaron una línea de investigación sobre cómo los docentes realizaban la docencia definiendo dos EE: el enfoque de transmisión de información centrado en el docente (ITTF) y el enfoque de transmisión centrado en el alumno (CCSF) (Jiménez, González y Tornel, 2020). Según diferentes investigadores los profesores que adoptan un enfoque ITTF centran su papel en transmitir información relacionada con los contenidos de aprendizaje y en las cuestiones técnicas del proceso de enseñanza (planifican cuidadosamente las clases, proporcionan resúmenes de los contenidos...) (Trigwell, Prosser y Waterhouse, 1999) mientras que en el enfoque CCSF el profesor concibe su enseñanza como facilitadora (Tezci, 2017) y tiene la intención de fomentar la creación de conocimiento favoreciendo la forma en cómo los estudiantes interpretan el mundo. En este sentido Trigwell y Prosser (1996) sugieren que el estudiante promueve el cambio conceptual dando lugar a un aprendizaje de alta calidad. En este enfoque el alumnado es el responsable último de la creación de conocimiento (Hynes, 2017).

Cabe destacar que Biggs y Moore (1993) realizaron una jerarquía en tres niveles que va de un enfoque cuantitativo, basado en la transmisión del conocimiento, seguido de un enfoque cualitativo donde el profesorado facilita la comprensión del significado hasta llegar a un nivel de cambio en el estudiante. Consideran que el tercer nivel es 'superior' a los niveles inferiores ya que los dos primeros niveles se centran en el docente y es el profesor el que controla el contenido y el uso de las destrezas docentes.

En este sentido, según Cañada (2012), el primer nivel centrado en el profesor busca la reproducción de los contenidos y la res- ponsabilidad de organizar o transformar el conocimiento es del profesor, quien también controla los contenidos.

Sin embargo, en el tercer nivel centrado en el estudiante, la función del docente es involucrar a los estudiantes en actividades de aprendizaje efectivo. Según Gargallo, Garfella, Sahuquillo, Verde y Jiménez (2015) hay una interacción bidireccional entre estudiante-profesor donde se promueve el trabajo cooperativo del alumno, necesario en su vida estudiantil y profesional.

Stes, Donche y Van Petegem (2014) indican que los resultados que se están obteniendo últimamente muestran un cambio hacia el enfoque CCSF en el que se deben tener en cuenta factores personales y un control del contexto. Por el contrario, un enfoque ITTF se asocia con una sensación de falta de control del contexto. Estos hallazgos subrayan la importancia del estudio de la enseñanza en relación con una variedad de factores personales y contextuales que influyen en la misma.

Cabe remarcar que los enfoques utilizados por los profesores pueden cambiar con el tiempo (Loredo, García y Cobo, 2017) y que son los alumnos los que valoran positivamente la docencia si esta se adapta a su forma de aprender (Tesouro, Corominas, Teixidó y Puiggalí, 2014).

\subsection{Estilos de aprendizaje}

Hay diferentes definiciones del significado de los EA. Hernández Pina y Hervás (2005) indican que "es un término acuñado para referir los procesos que surgen de las percepciones que los estudiantes tienen de las tareas académicas influenciadas por sus rasgos de personalidad" (p.285). Por su parte Abalde et al. (2001) hablan de EA haciendo referencia a "la forma en que los alumnos aprenden y estudian" (p. 465).

Otros autores como Corominas, Tesouro y Teixidó (2006) apuntan que estos EA potencian la predisposición del estudiante al aprender y la forma en cómo lo hace, es decir, se centran en el proceso, y no tanto en sus rasgos de personalidad, dando importancia a la percepción que se tiene de su situación particular o contexto.

En las investigaciones que se han llevado a cabo sobre el aprendizaje de los estudiantes hay teorías, como la de Kolb (1984), en la que se han definido cuatro enfoques, dependiendo de la experiencia de los alumnos: divergente, asimilador, convergente y acomodador. Como indican Martín y Rodríguez (2003), Kolb busca determinar y definir el concepto de reflexión. Otras investigaciones, como las de Marton y Saljo (1976), identificaron dos dependiendo de la forma en que estos aprenden (enfoque profundo y enfoque superficial). Otros estudios, como los llevados a cabo por el grupo de investigación de Entwistle y el grupo de Biggs, llegaron a conclusiones similares, aunque identificando tres enfoques (profundo, superficial y de alto rendimiento) que posteriormente, se simplificaron en dos. (Hernández Pina, García, Martínez, Hervás y Maquilón, 2002).

La información acerca de los EA puede ayudar a los profesores a ser más sensibles con las diferencias existentes entre los estudiantes en la clase y esto le permite procesos autorregulatorios del aprendizaje desde el conocimiento del estilo (García, Castañeda y Mansilla, 2018). Este conocimiento puede servir como una guía al diseñar las tareas de aprendizaje para que se relacionen con los EA, dependiendo del propósito del profesor (Alves, Miranda, Morais, Melaré, 2018).

Cabe destacar que existen diferentes opiniones respecto a la relación existente entre el estilo adoptado y el rendimiento académico. Estudios como los de Gutiérrez Tapias (2018), Muema, Mulwa y Mailu (2018), Ramudo, Barca, Brenlla, Peralbo y Barca (2020), entre otros, indican la existencia de dicha relación. Por el 
contrario, estudios como los de Kambani (2020), Vivas, Cabanillas y Vivas (2019)... no encontraron relación al respecto.

\subsection{Relaciones entre enfoques de enseñanza y estilos de aprendizaje}

Autores como Clara (2016) observaron que el EA del propio docente influencia sobre su EE, pero no de una forma directa, si bien la mayoría de estudios, como los de Hernández Pina y Monroy (2015), corroboran la asociación entre cómo los maestros enseñan y cómo aprenden los estudiantes. En esta línea Entwistle y Tait (1990) mostraron una evidencia sustancial en la influencia de los EE en los EA de los alumnos. Esta evidencia ha conllevado un interés científico creciente, como objeto de estudio, en los EE. Estos mismos autores observaron que las percepciones e interpretaciones del entorno académico de los estudiantes afectaban a sus EA de una forma directa y esto, a su vez, afecta a sus resultados de aprendizaje. En este sentido, también sugieren indagar acerca de las percepciones de los estudiantes y las preferencias sobre su entorno académico, en particular proponen investigar sobre la selección que haga el docente sobre las estrategias de enseñanza más apropiadas y en la estructuración del ambiente académico, cubriendo así mejor las necesidades de los estudiantes en su aprendizaje. El mismo Entwistle (1998) señala que los profesores deben proporcionar oportunidades para que los estudiantes aprendan de la manera que mejor se adapte a su estilo preferido de aprendizaje. Si los profesores adoptan un método de enseñanza demasiado extremo, quizás reflejando su propio estilo, se pueden encontrar con que un grupo de estudiantes utilice un enfoque ajeno a su forma de aprender.

Consecuentemente, debido a la escasez de investigaciones recientes en el contexto catalán, en el presente artículo se trata la percepción que tienen los estudiantes sobre la influencia de variables relacionadas con el entorno académico, para que sean tenidas en cuenta por el docente, así como también se reflexiona sobre la importancia del EE en relación con el EA.

\subsection{Objetivos}

- Elaborar y validar un cuestionario ad-hoc dirigido a estudiantes sobre EE y constatar su agrupación en dos factores (ítems centrados en el alumno e ítems centrados en el profesor) para que pueda ser utilizado en futuras investigaciones.

- Describir cómo influyen algunas variables contextuales (modalidad de estudio, curso, tipo de asignatura...) en el EE utilizado por el docente.

- Analizar si existe relación entre el EA utilizado por el alumno y el EE del docente.

\section{Método}

El presente trabajo se enmarca en la metodología cuantitativa no experimental de tipo descriptivo ya que se elaboró un instrumento cuantitativo y se dispuso de una muestra importante sobre la que se realizó un análisis estadístico en profundidad.

\subsection{Participantes}

La población fue de 13513 estudiantes matriculados en la Universidad de Girona y la muestra invitada a participar fue de 1850 estudiantes de diferentes facultades. Estos se clasificaron en tres grupos: a) Humanidades y Ciencias Sociales; b) Ciencias Naturales y de la Salud; c) Tecnología.

La muestra final, que contestó ambos instrumentos, fue de 1252 estudiantes (67.68\% de la muestra invitada). Cabe destacar que se trata de una muestra representativa con un margen de error máximo del $2.6 \%$ y con un intervalo de confianza del 95\%. En la tabla 1 se puede observar la composición en función de dos estratos (género y modalidad de estudio). La participación fue muy alta ya que diferentes profesores distribuyeron en sus propias aulas los dos cuestionarios juntos y en papel.

Tabla 1.

Distribución de la muestra

\begin{tabular}{|c|c|c|c|}
\cline { 2 - 3 } \multicolumn{1}{c|}{} & \multicolumn{2}{c|}{ Género } & \multirow{2}{*}{ Total } \\
\cline { 2 - 3 } & Masculino & Femenino & \\
\hline Humanidades y & 125 & 535 & 660 \\
Ciencias Sociales & $18.9 \%$ & $81.1 \%$ & $100.0 \%$ \\
\hline $\begin{array}{c}\text { Ciencias Naturales } \\
\text { y de la Salud }\end{array}$ & $38.8 \%$ & $61.2 \%$ & $100.0 \%$ \\
\hline & & & \\
\hline Tecnología & $80.8 \%$ & 145 & 355 \\
& & & $100.0 \%$ \\
\hline Total & 504 & 748 & 1252 \\
& $40.3 \%$ & $59.7 \%$ & $100.0 \%$ \\
\hline
\end{tabular}

La muestra es proporcional a la población, tal como se puede observar en la memoria de la propia universidad (UdG, 2019), ya que actualmente en Humanidades y Ciencias Sociales hay más estudiantes del género femenino, siendo a la inversa en el campo de la Tecnología. En Ciencias Naturales y de la Salud la diferencia entre el género masculino y femenino no es tan acentuada.

\subsection{Instrumento de recogida de información}

Se utilizaron dos cuestionarios con preguntas cerradas y escalas de Likert. Ambos se aplicaron conjuntamente si bien se añadieron algunas variables demográficas como género, modalidad de estudio, asignatura escogida por el alumno (teórica, práctica), curso y tipo de enfoque:

1. Para evaluar los EA se utilizó el apartado B-Approaches to studying (Tyler y Entwistle, 2007) que consta de 52 ítems del ASSIST y que evalúan el EA. Esta versión fue traducida al catalán y posteriormente validada (Tesouro, Cañabate y Puiggalí, 2014).

2. Basándonos en las diferentes características de los EE mencionadas en los estudios que hemos referenciado en la introducción se diseñó un cuestionario elaborado ad-hoc para evaluar el EE utilizado por el docente, desde el punto de vista del estudiante, presentado en una escala de Likert con valores entre 1 y 5 , siendo $1=$ siempre o casi siempre y $5=$ nunca o raramente. Se empezó a numerar por el ítem 53 ya que se presentó a continuación del cuestionario del apartado A que constaba de 52 ítems.

Este cuestionario consta de 14 ítems repartidos en dos factores (7 delimitan un enfoque centrado en el alumno mientras que los otros 7 delimitan un enfoque centrado en el profesor). No obstante, tal como se explica en el apartado del análisis factorial, se eliminaron 4 ítems y de esta forma se consiguió una mayor explicación de la varianza (64.1\%). 
Una vez elaborada la primera versión del instrumento de 14 ítems fue revisada y evaluada por dos expertos en Metodología de la Investigación. A partir de sus sugerencias se realizó una segunda versión mejorada del cuestionario.

En las tablas 2 y 3, se presenta la distribución de los ítems y se indican con un asterisco los que fueron eliminados para que sea tenido en cuenta en la aplicación del instrumento en futuras investigaciones.

Tabla 2.

Ítems que corresponden a un enfoque centrado en el alumno

\begin{tabular}{|c|l|}
\hline Ítem & \multicolumn{1}{c|}{ Contenido } \\
\hline 53 & $\begin{array}{l}\text { El/la profesor/a nos muestra su opinión sobre los temas } \\
\text { tratados y nos alienta a pensar por nosotros mismos, } \\
\text { quiere que elaboramos ideas propias. }\end{array}$ \\
\hline 54 & $\begin{array}{l}\text { Los exámenes que nos propone nos permiten expresar } \\
\text { a nuestra manera lo que hemos aprendido. }\end{array}$ \\
\hline 57 & $\begin{array}{l}\text { Nos enseña estrategias y maneras sobre cómo tenemos } \\
\text { que aprender y estudiar los conceptos o procedimien- } \\
\text { tos que nos explica al ir avanzando en los temas de la } \\
\text { asignatura. }\end{array}$ \\
\hline 58 & $\begin{array}{l}\text { Nos estimula a descubrir las evidencias que justifican } \\
\text { o dan soporte a diferentes puntos de vista. }\end{array}$ \\
\hline 60 & $\begin{array}{l}\text { Procura que analicemos críticamente la información } \\
\text { que nos proporciona. }\end{array}$ \\
\hline$* 61$ & $\begin{array}{l}\text { Se esfuerza en hacernos dar cuenta y en explicarnos } \\
\text { las relaciones y los vínculos entre la información y los } \\
\text { contenidos que nos proporciona. }\end{array}$ \\
\hline$* 62$ & $\begin{array}{l}\text { En sus clases incorpora espacios de participación y } \\
\text { discusión de los estudiantes sobre los temas tratados }\end{array}$ \\
\hline
\end{tabular}

Tabla 3.

Ítems que corresponden a un enfoque centrado en el alumno

\begin{tabular}{|c|c|}
\hline Ítem & \multicolumn{1}{c|}{ Contenido } \\
\hline 55 & $\begin{array}{c}\text { Sus clases son muy estructuradas y centradas en } \\
\text { los contenidos e información que quiere que apren- } \\
\text { damos. }\end{array}$ \\
\hline$* 56$ & $\begin{array}{l}\text { Los libros y lecturas que nos recomienda nos pro- } \\
\text { porcionan contenidos que van más allá de lo que nos } \\
\text { ha enseñado para ayudarnos a aprender por nuestra } \\
\text { cuenta. }\end{array}$ \\
\hline 59 & $\begin{array}{c}\text { El / la profesor/a nos dice exactamente lo que tene- } \\
\text { mos que anotar en el cuaderno de apuntes. }\end{array}$ \\
\hline 63 & $\begin{array}{c}\text { Su trabajo en esta asignatura se centra en transmi- } \\
\text { tir mucho de lo que él / ella sabe. }\end{array}$ \\
\hline 64 & $\begin{array}{c}\text { Los libros y lecturas que nos recomienda contienen } \\
\text { hechos, ejemplos o información sobre lo que él/ella ha } \\
\text { tratado en clase. }\end{array}$ \\
\hline
\end{tabular}

\begin{tabular}{|c|l|}
\hline Ítem & \multicolumn{1}{c|}{ Contenido } \\
\hline$* 65$ & $\begin{array}{c}\text { Nos hace comentarios y valoraciones favorables o } \\
\text { desfavorables del trabajo que hacemos individualmen- } \\
\text { te o en grupo para ayudar a mejorar las maneras de } \\
\text { estudiar y aprender }\end{array}$ \\
\hline 66 & $\begin{array}{l}\text { El/la profesor/a en las pruebas de evaluación nos } \\
\text { pide que reproduzcamos o expliquemos lo que hemos } \\
\text { aprendido de los apuntes, manuales o lecturas obli- } \\
\text { gatorias }\end{array}$ \\
\hline
\end{tabular}

\subsection{Procedimiento de recogida y análisis de datos}

Tal como se ha explicado en el apartado de la muestra, ambos instrumentos fueron distribuidos a la vez en formato papel a estudiantes de diferentes titulaciones y se les indicó que, antes de empezar a contestarlos, pensaran en una asignatura concreta para poder detectar si existían diferencias en función del tipo de asignatura (teórica, práctica o teórica y práctica a la vez). También se le presentaron los objetivos del trabajo y se les garantizó la confidencialidad. Finalmente, los datos fueron analizados con los programas SPSSx v.27 y AMOS v.25 y se realizó un análisis factorial exploratorio del cuestionario ad-hoc, estadíticos descriptivos, chi cuadrado....

\section{Resultados}

\subsection{Análisis factorial del cuestionario sobre enfoques de enseñanza utilizados}

Se realizó un análisis factorial del cuestionario ad-hoc sobre EE, a fin de confirmar la asociación entre los dos factores (ítems centrados en el alumno e ítems centrados en el profesor). En este análisis se utilizó una técnica oblicua (Oblimin), debido a que en el análisis confirmatorio se observó una correlación entre los factores propuestos. Tal como se ha comentado en el apartado de instrumentos, se observó que existían cuatro ítems que presentaban incongruencias (ítems 56, 61, 62 y 65) y fueron eliminados ya que si se mantenían sólo se explicaba el 55.02 \% de la varianza.

Seguidamente se volvió a realizar el análisis factorial exploratorio (Tabla 4 y Figura 1) y los valores obtenidos de la esfericidad de Barlet (superior a .8) y KMO (valor elevado y con $\mathrm{p}=.00$ ) nos indicaron que la estructura de los datos era adecuada para ser analizada factorialmente. Los ítems se agruparon en 2 factores: en el primer factor los 5 ítems que hacen referencia al enfoque centrado en el alumno $(53,54,57,58$ y 60$)$ y en el segundo los 5 centrados en el profesor $(55,59,63,64$ y 66$)$ y se explica el $64.09 \%$ de la varianza.

Tabla 4.

Análisis factorial exploratorio

\begin{tabular}{|c|c|c|}
\hline \multicolumn{3}{|c|}{ Prueba de KMO y Bartlett } \\
\hline Medida Kaiser-Meyer-Olkin de adecuación de muestreo &, 880 \\
\hline \multirow{2}{*}{ Prueba de esfericidad de Bartlett } & Aprox. Chi-cuadrado & 5603,935 \\
\cline { 2 - 3 } & gl & 45 \\
\cline { 2 - 3 } & Sig. &, 000 \\
\hline
\end{tabular}




\begin{tabular}{|c|c|c|c|c|c|c|c|c|c|}
\hline \multicolumn{10}{|c|}{ Varianza total explicada } \\
\hline \multirow[b]{2}{*}{ 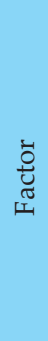 } & \multicolumn{3}{|c|}{$\begin{array}{c}\text { Autovalores } \\
\text { iniciales }\end{array}$} & \multicolumn{3}{|c|}{$\begin{array}{c}\text { Sumas de cargas } \\
\text { al cuadrado } \\
\text { de la extracción }\end{array}$} & \multicolumn{3}{|c|}{$\begin{array}{c}\text { Sumas de cargas } \\
\text { al cuadrado } \\
\text { de la rotación }\end{array}$} \\
\hline & $\underset{\tilde{0}}{\overline{0}}$ & 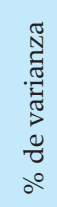 & 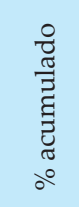 & సٓㅠㅇ & 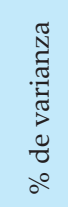 & 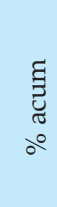 & 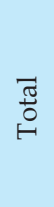 & 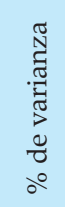 & 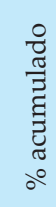 \\
\hline 1 & 4,3 & 43,3 & 43,31 & 3,9 & 38,8 & 38,8 & 3,0 & 29,9 & 29,9 \\
\hline 2 & 2,1 & 20,8 & 64,09 & 1,7 & 16,7 & 55,5 & 2,6 & 25,5 & 55,5 \\
\hline 3 & 6 & 6,2 & 70,3 & & & & & & \\
\hline 4 & 6 & 5,6 & 76 & & & & & & \\
\hline 5 &, 5 & 5,4 & 81,3 & & & & & & \\
\hline 6 &, 5 & 4,7 & 86,0 & & & & & & \\
\hline 7 & ,4 & 4,4 & 90,4 & & & & & & \\
\hline 8 &, 3 & 3,6 & 93,9 & & & & & & \\
\hline 9 & ,3 & 3,4 & 97,3 & & & & & & \\
\hline 10 & , & 2,6 & 100,0 & & & & & & \\
\hline
\end{tabular}

Método de extracción: máxima verosimilitud.

\begin{tabular}{|c|c|c|}
\hline \multicolumn{3}{|c|}{ Prueba de bondad de ajuste } \\
\hline Chi-cuadrado & gl & Sig. \\
\hline 107,832 & 26 &, 000 \\
\hline
\end{tabular}

\begin{tabular}{|l|c|c|}
\hline \multirow{3}{*}{} & \multicolumn{2}{|c|}{ Matriz de factor rotado ${ }^{2}$} \\
\cline { 2 - 3 } & 1 & 2 \\
\hline Ítem 53 &, 825 & \\
\hline Ítem 54. &, 778 &, 648 \\
\hline Ítem 55 & &, 636 \\
\hline Ítem 57 &, 659 &, 794 \\
\hline Ítem 58 &, 872 &, 771 \\
\hline Ítem 59 & \multicolumn{2}{|c|}{} \\
\hline Ítem 60 & &, 677 \\
\hline Ítem 63 & & \\
\hline Ítem 64 & & \\
\hline Ítem 66 & & \\
\hline $\begin{array}{l}\text { Método de extracción: máxima verosimilitud. } \\
\text { Método de rotación: Varimax con normalización Kaiser. } \\
\text { a. La rotación ha convergido en 3 iteraciones. }\end{array}$ \\
\hline
\end{tabular}

El valor del Alfa de Cronbach (.80) nos indica una fiabilidad aceptable. Para descartar la unidimensionalidad de la escala se realizó un análisis factorial forzando a un factor y se vio que sólo se explicaba un $43.31 \%$ de la varianza. Los valores del análisis factorial exploratorio y los índices de ajuste del confirmatorio son adecuados y válidos, lo que denota que el instrumento elaborado ad-hoc dispone de unas adecuadas propiedades psicométricas.

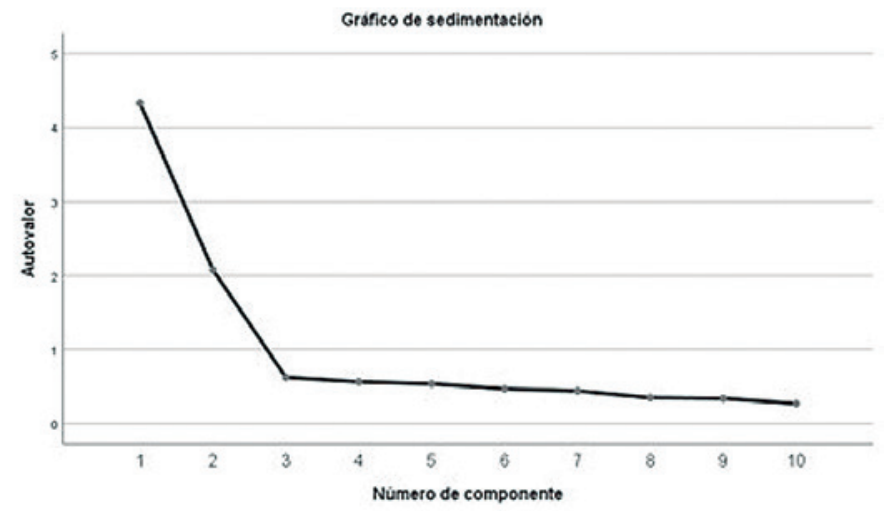

Figura 1. Análisis factorial (gráfico de sedimentación)

Para realizar el análisis factorial confirmatorio se utilizó la matriz de covarianzas al tratarse de datos procedentes de una escala de Likert. Los resultados obtenidos en el ajuste al modelo por medio de las cargas factoriales (Figura 2 y Tabla 5) indicaron ser aceptables: $\chi 2(1252)=165.84 ; \mathrm{p}<.00 ; \chi 2 / \mathrm{df}=4.88 ; \mathrm{CFI}=.98$; RMSEA=.56; TLI=.97; PRATIO=.76; PNFI=.73; PCFI=.74, CMIN/ $\mathrm{DF}=4.88$.

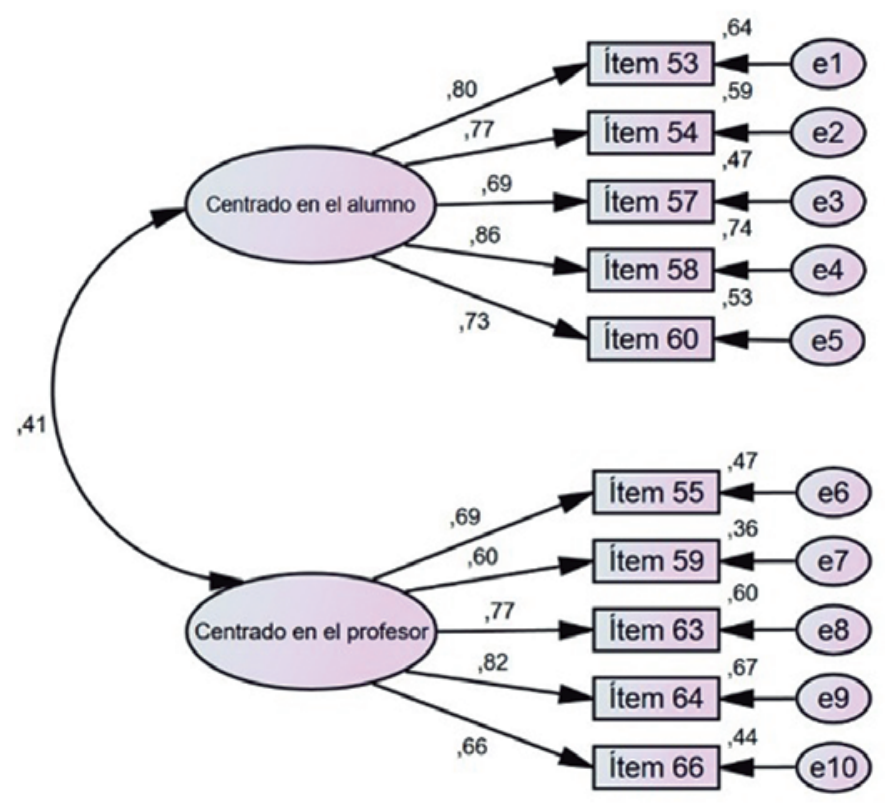

Figura 2. Ajuste al modelo por medio de las cargas factoriales 
Tabla 5 .

Fiabilidad a través del Coeficiente alfa de Cronbach

\begin{tabular}{|c|c|c|}
\hline \multicolumn{3}{|c|}{ Estadísticas de fiabilidad } \\
\hline $\begin{array}{c}\text { Alfa } \\
\text { de Cronbach }\end{array}$ & $\begin{array}{c}\text { Alfa de Cronbach basada } \\
\text { en elementos estandarizados }\end{array}$ & $\begin{array}{c}\text { N. }{ }^{\circ} \\
\text { de elementos }\end{array}$ \\
\hline, 851 &, 852 & 10 \\
\hline
\end{tabular}

\subsection{Resultados obtenidos por el cuestionario de EE}

El enfoque centrado en el alumno obtuvo una media ligeramente superior al centrado en el profesor siendo ambas representativas. Los valores de las curtosis y las asimetrías nos indicaron que las puntuaciones tienden a ser ligeramente dispersas y relativamente bajas (tabla 6).

Tabla 6

Estadísticos de los EE

\begin{tabular}{|c|c|c|c|c|}
\cline { 2 - 5 } \multicolumn{1}{c|}{} & $\bar{x}$ & $\sigma$ & Curtosis & Asimetría \\
\hline $\begin{array}{c}\text { Centrado } \\
\text { en el alumno }\end{array}$ & 3.16 & .96 & -.18 & -.60 \\
\hline $\begin{array}{c}\text { Centrado } \\
\text { en el profesor }\end{array}$ & 3.03 & .99 & -.17 & -.51 \\
\hline
\end{tabular}

Se aplicó la prueba $\chi 2$ para ver si se generaban diferencias entre los dos EE y las variables contextuales y se observaron que existían diferencias significativas en la modalidad de estudio, género, tipo de asignatura y curso (Tabla 7).

Observamos que la modalidad de estudio afecta en el enfoque de aprendizaje utilizado por el profesor $(p=.00)$. Los estudios de Humanidades y Ciencias Sociales tienden a utilizar un EE centrado en el alumno mientras que los de Tecnología y Ciencias Naturales y de la Salud tienden a utilizar un EE centrado en el docente.

En el caso del género del profesorado observamos que, en general, las mujeres adoptan un enfoque más centrado en el alumno mientras que los hombres adoptan un enfoque más centrado en el profesor $(p=.00)$. Si diferenciamos las modalidades por género vemos que las mujeres $(p=.00)$ en las titulaciones de humanidades escogen un enfoque centrado en el alumno mientras que en las científicas y tecnológicas utilizan el centrado en el profesor $(p=.00)$. En el caso del género masculino en las titulaciones de humanidades y ciencias de la naturaleza eligen un enfoque centrado en el alumno y en las tecnológicas utilizan el centrado en el profesor $(p=.00)$.

Tabla 7.

Relación entre las variables contextuales (modalidad de estudio, género, tipo de asignatura y curso) y tipo de enfoque de enseñanza

\begin{tabular}{|c|c|c|c|}
\hline Variable contextual & $\begin{array}{c}\text { Sig. } \\
\text { bilateral }\end{array}$ & $\begin{array}{c}\text { Grado } \\
\text { de libertad }\end{array}$ & $\begin{array}{c}\text { Chi-cuadrado } \\
\text { de Pearson }\end{array}$ \\
\hline $\begin{array}{c}\text { Modalidad de estu- } \\
\text { dio (Humanidades y } \\
\text { Ciencias Sociales vs } \\
\text { Tecnología y Ciencias } \\
\text { Naturales y de la } \\
\text { Salud) }\end{array}$ & $.00^{*}$ & 2 & 68.751 \\
\hline Género & $.00^{*}$ & 1 & 14.703 \\
\hline
\end{tabular}

\begin{tabular}{|c|c|c|c|}
\hline Variable contextual & $\begin{array}{c}\text { Sig. } \\
\text { bilateral }\end{array}$ & $\begin{array}{c}\text { Grado } \\
\text { de libertad }\end{array}$ & $\begin{array}{c}\text { Chi-cuadrado } \\
\text { de Pearson }\end{array}$ \\
\hline Curso $\left(1 . .^{\circ}, .^{\circ}, 3 .{ }^{\circ}\right.$ y $\left.4 . .^{\circ}\right)$ & $.00^{*}$ & 4 & 84.612 \\
\hline $\begin{array}{c}\text { Tipo de asignatura } \\
\text { (teórica o práctica) }\end{array}$ & $.01^{*}$ & 2 & 10.085 \\
\hline
\end{tabular}

En cambio, el tipo de asignatura sí que afecta ( $\mathrm{p}=.01)$. El profesor adopta un enfoque centrado en el alumno cuando la asignatura es práctica mientras que, por el contrario, escoge un enfoque más centrado en el profesor cuando se trata de asignaturas teóricas.

Respecto al curso en el que se imparte la asignatura, los alumnos de $1^{\circ}$ y $2^{\circ}$ curso indican que los profesores presentan un enfoque centrado en el alumno. En cambio, los de $3^{\circ}$ y $4^{\circ}$ curso muestran una tendencia a un enfoque centrado en el profesor $(\mathrm{p}=.00)$.

3.3.Resultados obtenidos por el cuestionario de estilos de aprendizaje

Del análisis de cuestionario de EA (Tabla 8) se extrae que la muestra presenta un enfoque preferentemente profundo $(\bar{x}=3.31)$, seguido por el estratégico $(\bar{x}=3.28)$ y por el superficial $(\bar{x}=3.04)$.

Tabla 8.

Resultados del ASSIST

\begin{tabular}{|l|c|c|c|c|}
\hline & & & & \\
Escala/subescala & & & $\bar{x}$ & $\frac{0}{5}$ \\
\hline Profundo & 3.31 & .54 & -.21 & .14 \\
\hline Búsqueda de significado & 3.50 & .65 & -.41 & .30 \\
\hline Relación de ideas & 3.14 & .73 & -.09 & .17 \\
\hline Uso de evidencia & 3.48 & .67 & -.35 & -.00 \\
\hline Interés en las ideas & 3.11 & .73 & -.22 & -.10 \\
\hline Estratégico & 3.28 & .57 & -.21 & -.08 \\
\hline Estudio organizado & 3.39 & .83 & -.37 & -.24 \\
\hline Gestión del tiempo de estudio & 3.01 & .86 & -.03 & -.54 \\
\hline Alerta a las demandas en evaluaciones & 3.79 & .69 & -.51 & -.17 \\
\hline Logro & 3.50 & .72 & -.37 & .03 \\
\hline Monitoriza efectividad & 3.60 & .72 & -.40 & .05 \\
\hline Superficial & 3.04 & .49 & .02 & .08 \\
\hline Falta de propósito & 2.85 & .80 & .05 & -.35 \\
\hline Memorización sin relación & 2.74 & .67 & .13 & -.13 \\
\hline Limitado al syllabus & 3.32 & .70 & .02 & -.23 \\
\hline Miedo al fracaso & 3.26 & .75 & -.17 & -.19 \\
\hline
\end{tabular}

Los valores de las desviaciones típicas son muy pequeñas, tanto en las clases como de las subclases, y esto nos indica que las puntuaciones se agrupan alrededor de la media. Si observamos los valores de la asimetría en las clases existen dos valores negativos (enfoques profundo y estratégico) lo cual nos confirma que dichos enfoques presentan puntuaciones algo más altas. Finalmente, el valor de la curtosis nos indica que existe una agrupación ligeramente superior de las puntuaciones en el enfoque profundo y estratégico. 
3.4.Influencia del estilo de aprendizaje del alumno en el enfoque de enseñanza del profesor

Si partimos de los resultados obtenidos en el cuestionario para evaluar los EA de Tyler y Entwistle (2007) y los relacionamos con los obtenidos en el cuestionario ad-hoc, sobre los EE utilizado por el docente desde el punto de vista del estudiante, aplicando el $\chi 2$, vemos que existe una fuerte relación entre las variables EA y EE $(\mathrm{p}=.00)$ (Tabla 9).

Tabla 9.

Relación entre estilo de aprendizaje y tipo de enfoque de enseñanza

\begin{tabular}{|c|c|c|c|c|c|c|}
\hline & & & Estil & e Apre & izaje & \\
\hline & & & 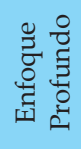 & 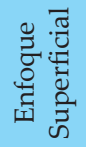 & 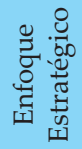 & Е \\
\hline \multirow{6}{*}{ 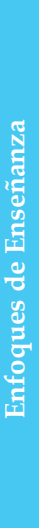 } & \multirow{2}{*}{ 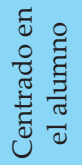 } & Recuento & 289.0 & 162.0 & 230.0 & 681.0 \\
\hline & & $\begin{array}{l}\text { Recuento } \\
\text { esperado }\end{array}$ & 262.7 & 194.7 & 223.6 & 681.0 \\
\hline & \multirow{2}{*}{ 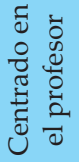 } & Recuento & 194.0 & 196.0 & 181.0 & 571.0 \\
\hline & & $\begin{array}{l}\text { Recuento } \\
\text { esperado }\end{array}$ & 220.3 & 163.3 & 187.4 & 571.0 \\
\hline & \multirow{2}{*}{$\underset{⿱ 乛 \pi}{0}$} & Recuento & 483.0 & 358.0 & 411.0 & 1252.0 \\
\hline & & $\begin{array}{l}\text { Recuento } \\
\text { esperado }\end{array}$ & 483.0 & 358.0 & 411.0 & 1252.0 \\
\hline
\end{tabular}

Por una parte, los alumnos que presentan un EA profundo indican que el profesor presenta un enfoque centrado en el alumno. Por otra parte, los alumnos, cuyo estilo es superficial, señalan que el enfoque utilizado por el docente es mayoritariamente el centrado

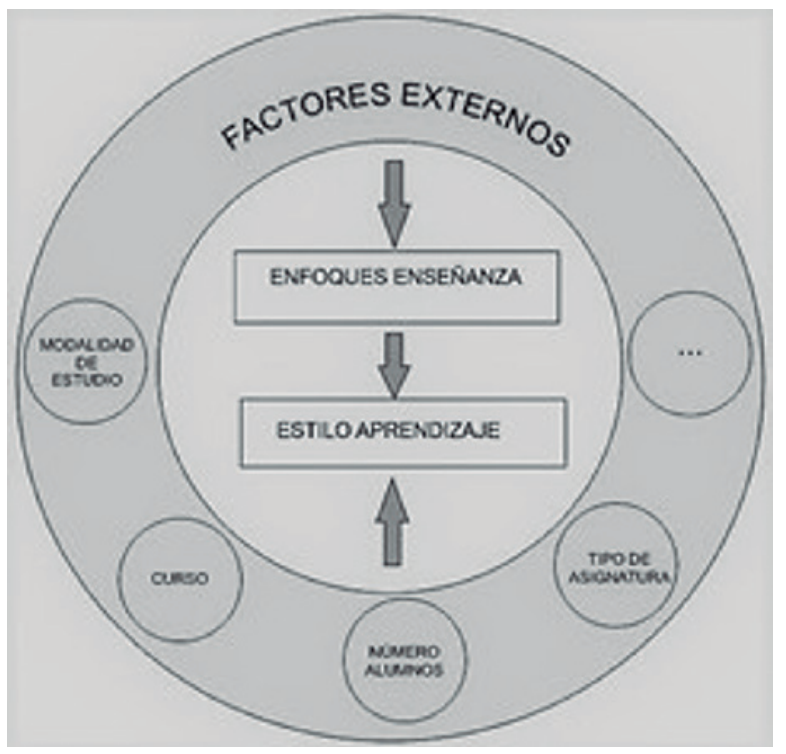

Figura 3. Influencias en los EE y EA en el profesor $y$, finalmente, los que utilizan un enfoque estratégico se decantan levemente por un enfoque centrado en el alumno.

En general se corrobora que hay una serie de factores externos (tipo de asignatura, curso en que se imparte...) que influyen en la elección por parte del docente del EE utilizado. También se observa que el EE utilizado por el profesor influye en el estudiante en la adopción de su EA (Figura 3).

\section{Discusión}

El primer objetivo de crear un cuestionario para estudiantes sobre EE (centrado en el alumno y centrado en el profesor) se ha conseguido y puede ser de gran utilidad en futuras investigaciones.

En relación con el segundo objetivo, que consistía en observar si existen variables contextuales que afectan a la elección por parte del docente del EE utilizado, en este estudio se ha observado la repercusión que tiene la modalidad de estudio en el EE del profesor. Tal como hemos apuntado, en los estudios de Humanidades y Ciencias Sociales los profesores tienden a utilizar un EE centrado en el alumno, mientras que los de Tecnología y Ciencias Naturales y de la Salud tienden a utilizar un EE centrado en el docente. Cabe destacar que el tipo de contenidos que se imparten en los distintos grados son muy diferentes, así como también lo son las características de los estudiantes, por lo que el docente adopta diferentes estilos de enseñanza. En las titulaciones del ámbito Humanístico y Social los contenidos están más orientados a la compresión y reflexión por lo que se requieren de una mayor ayuda por parte del profesor para hacer una asimilación e interpretación correcta de los mismos. En cambio, en el ámbito tecnológico los contenidos, al ser más objetivos, no requieren tanto de un profesor centrado en el estudiante. Esto estaría acorde con los resultados obtenidos por Iqbal, Saeed, Abiodullah, y Khatoon (2013).

Con relación al tipo de profesión cabe destacar que para desarrollar con éxito una profesión humanística o social es muy importante el ejemplo de los profesores y, por lo tanto, en muchas materias puede ser más importante la forma de actuar de un profesor que los propios contenidos. Estos resultados van en la misma línea a los obtenidos por Feixas (2010) que también observó que los profesores de ciencias humanas y ciencias sociales utilizaban más un enfoque centrado en el alumno y en las ciencias experimentales y ciencias de la salud optaban por un enfoque centrado en el profesor.

Contrariamente, Emanuel y Potter (1992) describen su sorpresa por la falta de grandes diferencias de preferencias de estilo entre las diferentes carreras universitarias.

Respecto a la variable género, tal como hemos observado en el apartado de resultados, son las profesoras las que adoptan un enfoque más centrado en el alumno.

En relación con la influencia que tiene la asignatura escogida en el tipo de enfoque observamos que los profesores que imparten una asignatura mayoritariamente práctica suelen utilizar un enfoque centrado en el alumno y al revés sucede en las asignaturas teóricas. Cabe señalar que en que en las asignaturas prácticas el estudiante necesita un mayor acompañamiento por parte del profesor para poder poner en práctica los contenidos teóricos aprendidos y desarrollar un aprendizaje competencial mientras que en las asignaturas teóricas los estudiantes no necesitan tanto la ayuda del profesor y pueden trabajar más autónomamente.

Así mismo encontramos diferencias estadísticamente significativas con la variable curso. Cuanto más bajo es el curso más considera el estudiante que el profesor presenta un enfoque centrado en el alumno ya que en los primeros cursos el estudiante necesita un período de adaptación y requiere un mayor acompañamiento 
por parte de sus profesores. Contrariamente, cuanto más alto es el curso más considera el estudiante que el profesor está centrado en transmitir contenidos porque, al haber superado la fase de adaptación, ya dispone de una base y autonomía suficiente para poder asimilar y profundizar los contenidos sin ayuda del profesorado.

Respecto al tercer objetivo se observa que existe relación entre el EA utilizado por el estudiante y el EE del profesor. En los resultados obtenidos se advierte que los alumnos cuyos profesores adoptan un EE centrado en el profesor tienden a adoptar un estilo superficial. Cuando el alumno percibe que su profesor muestra interés, no sólo por transmitir contenidos, sino también en atender a la diversidad y muestra a sus estudiantes situaciones auténticas y funcionales y se ocupa de qué resultados de aprendizaje logran sus estudiantes, genera en éstos una actitud más positiva que a menudo genera la adopción de un EA más profundo. Esto estaría acorde con lo indicado por otros autores como Prosser et al. (2003), Rosario et al. (2014)... En esta línea Rosário et al. (2013) concluye que en su investigación el EA profundo correlacionó positivamente con el enfoque centrado en el alumno y negativamente con el enfoque centrado en el profesor. Este hallazgo es importante y puede contribuir a alentar a los maestros a tomar más conciencia de los EA de sus alumnos y a reflexionar sobre la efectividad de sus propios métodos de enseñanza.

También en nuestras aulas observamos que los estudiantes aprenden mejor si lo que se les explica les interesa y está vinculado a su contexto, es decir, si se propicia un aprendizaje significativo para ellos y tiene que ver con su futura profesión; por lo tanto, el EE del profesor debería ayudar a construir competencias y no centrarse tanto ni en los contenidos como referente último del aprendizaje ni en una evaluación final. Cabe destacar que estos resultados coinciden con los obtenidos por Trigwell et al. (1999) que consideran que el profesor que dedica más tiempo a interactuar con los alumnos solucionando sus dudas, realizando debates... hace que éstos tiendan a no utilizar un enfoque superficial.

En un estudio previo realizado por Tesouro y Puiggalí (2016), con 259 profesores de la Universidad de Girona, se extrajeron resultados similares a los obtenidos en este estudio realizado con estudiantes. Según los docentes, existía relación respecto al ámbito de estudio. Se observó que en Humanidades/Educación (Letras, Educación y Psicología) el profesor utilizaba un enfoque más centrado en el alumno mientras que en Ciencias Sociales Derecho, Económicas/Empresariales, Turismo y Ciencias/Tecnología el profesorado presentaba un enfoque más centrado en el profesor o central.

Finalmente, se puede concluir que el profesor no utiliza siempre el mismo tipo de enfoque, sino que éste se puede adaptar también a factores externos como el tipo de asignatura que está impartiendo y a otros factores como pueden ser el número de alumnos, curso en que se imparte la asignatura, etc.

También es importante remarcar que se ha creado un instrumento de medición nuevo más breve, práctico para su aplicación y con unas propiedades psicométricas adecuadas. Este podría ser de gran utilidad para conocer el tipo de EE con la finalidad de mejorar la práctica docente en la Educación Superior.

A partir de este estudio, como posibles sugerencias, nos planteamos poder aplicar los instrumentos mencionados en diferentes universidades, tanto del territorio nacional como internacional, y así poder aumentar la muestra debido a que el contexto de la intervención ha sido limitado. También sería interesante poder pasar un cuestionario a los profesores para poder triangular los resultados con los obtenidos de sus propios alumnos debido a que en esta investigación solo hemos recogido las opiniones de los estudiantes.

\section{Referencias}

Abalde, E., Muñoz, M., Buendia, L., Olmedo, E. M., Berrocal, E., Cajide, J. ... Maquilon, J. (2001). Los enfoques de aprendizaje en estudiantes universitarios españoles. Revista de Investigación Educativa, 19(2), 465-489. Recuperado de https://bit.ly/3adIC66

Alves, P., Miranda, L., Morais, C. y Melaré, D. (2018). Estilos de aprendizaje de los estudiantes de la educación superior y el acceso a las herramientas de entornos virtuales. Tendencias Pedagógicas, 31, 69-81. doi: https://bit.ly/2OsDoep

Biggs, J.B. y Moore, P.J. (1993). Process of learning (3rd ed). Sydney: Prentice Hall. Recuperado de https://bit.ly/3jIzRUN

Cañada, M.D. (2012). Enfoque docente de la enseñanza y el aprendizaje de los profesores universitarios y usos educativos de las TICS. Revista de Educación, 359, 388-412. doi: 10.4438/1988592X-RE-2011-359-099

Clara, A. (2016). ¿Enseño como aprendí?: el rol del estilo de aprendizaje en la enseñanza del profesorado universitario. Aula Abierta, 44(02), 91-98. Recuperado de https://bit.ly/3788q1I

Corominas, E., Tesouro, M. y Teixidó, J. (2006). Vinculación de los enfoques de aprendizaje con los intereses profesionales y los rasgos de personalidad. Aportaciones a la innovación del proceso de enseñanza y aprendizaje en la educación superior. Revista de investigación Educativa, 24(2), 443-473. Recuperado de https://bit.ly/2LJmzuK

Emanuel, R.C. y Potter,W.J. (1992). Do students' style preferences differ by grade level, orientation toward college and academic major? Research in Higher Education, 33(3), 394-414. Recuperado de https://bit.ly/3ac5t1V

Entwistle, N.J. (1998). Styles of learning and teaching: An integrated outline of educational psychology for students, teachers and lecturers. New York: Routledge.

Entwistle, N.J. y Tait, H. (1990). Approaches to learning, evaluations of teaching, and preferences for contrasting academic environments. Higher Education, 19(2), 169-194. Recuperado de https://bit.ly/3qghARn

Feixas, M. (2010). Enfoques y concepciones docentes en la universidad. RELIEVE, 16(2), 1-27. Recuperado de https://bit. ly/3d $82 X v u$

Frenzel, A.C. (2014). Teacher emotions. En E. A. Linnenbrink-Garcia y R. Pekrun (Eds.), International Handbook of Emotions in Education (pp. 494-519). New York: Routledge.

García, C., Castañeda, E. y Mansilla, J.M. (2018). Experiencia de innovación en el aula desde la autorregulación y los estilos de aprendizaje. Tendencias Pedagógicas, 31, 137-148. doi: https:// bit.ly/3qgxuLw

Gargallo, B., Garfella, P., Sahuquillo, P., Verde, I. y Jiménez, M.A. (2015). Métodos centrados en el aprendizaje, estrategias y enfoques de aprendizaje en estudiantes universitarios. Revista de Educación, 370, 229-254. Recuperado de https://bit.ly/2Nor0LP

Gutiérrez Tapias, M. (2018). Estilos de aprendizaje, estrategias para enseñar. su relación con el desarrollo emocional y “aprender a aprender". Tendencias Pedagógicas, 31, 83-96. doi: 10.15366/tp2018.31.004

Hernández Pina, F., García, M.P., Martínez, P., Hervás, R.M. y Maquilón, J. (2002). Consistencia entre motivos y estrategias de aprendizaje en estudiantes universitarios. Revista de Investigación Educativa, 20(2), 487-510. Recuperado de https://bit. ly/3qhP6Xg

Hernandez Pina, F. y Hervás, R.M. (2005). Enfoques y estilos de aprendizaje en Educación Superior. REOP, 16(2), 283-299. doi: https://bit.ly/2NmG1xI

Hernández Pina, F. y Monroy, F.A. (2015). A preliminary sudy of teachers' perception of core competencies for undergra- 
duate students. Psicología Educativa, 21(1), 11-16. doi: https:// bit.ly/2MZnsju

Hynes, M. (2017). Students-as-producers: Developing valuable student-centered research and learning opportunities. International Journal of Research Studies in Education, 7(4), 1-13. doi: 10.5861/ijrse.2017.1858

Iqbal, T., Saeed, A., Abiodullah, M. y Khatoon, M. (2013). Relationship of Students Approaches to Studying and Teachers. Approaches to Teaching at Graduate Level in Punjab Pakistan. Secondary Education Journal, 2(1), 1-17. Recuperado de https:// bit.ly/3b3VM5b

Jiménez, D., González, J.J. y Tornel, M. (2020). Metodologías activas en la universidad y su relación con los enfoques de enseñanza. Profesorado. Revista de Currículum y Formación de Profesorado, 24(1), 76-94. doi: 10.30827/profesorado.v24i1.8173

Kambani, A. (2020). Teaching Methods and Students. Academic Performance in Kinematical Motion: Graphical Interpretation and Conceptual Understanding. American Journal of Social Sciences and Humanities, 5(1): 69-103. doi: 10.20448/801.51.69.103

Kolb, D.A. (1984). Experimential Learning. Englewood Cliffs, Nueva Jersey: Prentice Hall.

Loredo, J.R., García, B. y Cobo, K.M. (2017). Estilos de enseñanza de los profesores, un estudio sobre la planeación, conducción y evaluación que realizan en el aula. Tendencias Pedagógicas, 30, 17-36. doi: https://bit.ly/3jURQYx

Martín, A. y Rodríguez, M.J. (2003). Estilos de aprendizaje y grupos de edad: comparación de dos muestras de estudiantes jóvenes y mayores. Aula Abierta, 82, 97-114

Marton, F. y Säljö, R. (1976). On qualitative differences in learning. I. Outcome and process. British Journal of Educational Psychology, 46, 4-11. doi: https://bit.ly/378FoiF

Muema, J., Mulwa, D. y Mailu, S. (2018). Relationship between teaching method and Students'Performance in mathematics in public secondary schools In Dadaab Sub County, Garissa County; Kenya. IOSR Journal of Research E Method in Education, 8(5),59-63. doi: 10.9790/7388-0805055963

Özyurt, Ö. y Özyurt, H. (2015). Learning style based individualized adaptive e-learning environments: Content analysis of the articles published from 2005 to 2014. Computers in Human Behavior, 52, 349-358. doi: https://bit.ly/2ZdAHiV

Prosser, M. y Trigwell, K. (2006). Confirmatory factor analysis of the Approaches to Teaching Inventory. British Journal of Educational Psychology, 76, 405-419. doi: 10.1348/000709905X43571

Prosser, M., Ramsden, P., Trigwell, K. y Martin, E. (2003). Dissonance in experience of teaching and its relation to the quality of student learning. Studies in Higher Education, 28, 37- 48. doi: https://bit.ly/3rKLqxG

Ramudo, I., Barca, E., Brenlla, J.C., Peralbo, M., y Barca, A. (2020). Predicción del rendimiento académico del alumnado de Bachillerato: efecto de los enfoques de aprendizaje y atribuciones causales. Revista de Psicología y Educación, 15(2), 108-120. doi: https://bit.ly/2Ze3ZOq

Rosário, P., Núñez, J., Valle, A., Paiva, O. y Polydoro, S. (2013). Approaches to Teaching in High School when Considering Contextual Variables and Teacher Variables. Revista de Psicodidáctica, 18(1), 25-45. Recuperado de https://bit.ly/3jLeORq
Rosário, P., Núñez, J., Vallejo, G., Paiva, O., Valle, A., Fuentes, S. y Pinto, C. (2014). Are teachers' approaches to teaching responsive to individual student variation? A two-level structural equation modelling. European Journal of Psychology of Education, 29(4), 577-601. doi:10.1007/s10212-014-0214-9.

Soflano, M., Connolli, T.M. y Haney, T. (2015). Learning style analysis in adaptive GBL application to teach SQL. Computers $\mathcal{E}$ Education, 86, 105-119. doi: https://bit.ly/3b5JY2m

Stes, A., Donche, V. y Van Petegem, P. (2014). Understanding differences in teaching approaches in higher education: An evidence-based perspective. Reflecting Education, 9 (1), 21-35. Recuperado de https://bit.ly/3rM5Mqj

Stes, A., Gijbels, D. y Van Petegem, P. (2008). Student-focused approaches to teaching in relation to context and teacher characteristics. Higher Education, 55, 255-267. doi: https://bit. ly/2Z9Mejx

Tezci, E. (2017). Adaptation of ATI-R Scale to Turkish Samples: Validity and Reliability Analyses. International Education Studies, 10(1), 67-81. doi: org/10.5539/ies.v10n1p67

Tesouro, M., Cañabate, D. y Puiggalí, J. (2014). Los enfoques de aprendizaje en estudiantes universitarios catalanes mediante el Approaches and Study Skills Inventory for Students (assist). Revista de Investigación Educativa, 32(2), 479-498. doi: https:// bit.ly/3qhQSro

Tesouro, M., Corominas, E., Teixidó, J. y Puiggalí, J. (2014). La autoeficacia docente e investigadora del profesorado universitario: relación con su estilo docente e influencia en sus concepciones sobre el nexo docencia-investigación. Revista de Investigación Educativa, 32(1), 169-186. doi: https://bit.ly/3jJTbkv.

Tesouro, M. y Puiggalí, J. (2016). La influencia de los factores contextuales y personales del docente en los enfoques de enseñanza utilizados. Revista del CIDUI, 3. 1-11. Recuperado de https://bit.ly/3jHzxWm

Trigwell, K. y Prosser, M. (1996). Congruence between intention and strategy in science teachers'approach to teaching. Higher Education, 32, 77-87. Recuperado de https://bit.ly/2LLdYYB

Trigwell, K., Prosser, M. y Waterhouse, F. (1999). Relations between teachers' approaches to teaching and students' approaches to learning. Higher Education, 37(1), 57-70. doi: https://bit. ly/3pcMzfH

Tyler, S. y Entwistle, N. J. (2007). Approaches to learning and studying inventory: self-score version (3rd edition). En: S. Tyler (Ed.), The Managers Good Study Guide: an esential reference with key concepts, tools and techniques explained. Open University's good study guide series. (pp. 339-352). Milton Keynes: Open University Worldwide Ltd.

UdG (2019). UdG Impacte. Curs acadèmic 2018-19. Recuperado de https://bit.ly/3d3HST7

Vivas, R. Cabanilla, E. y Vivas, W. (2019). Relación entre los estilos de aprendizaje y el rendimiento académico del estudiantado de la carrera de Ingeniería Agronómica de la Universidad Central del Ecuador. Revista Educación, 43 (1), 1-15. doi: https://bit.ly/2Z9x5hQ 
